\title{
Editorial
}

\section{On the cusp of change or chimera?}

Asian Business \& Management (2010) 9, 1-3. doi:10.1057/abm.2009.27

Human history has witnessed a succession of social institutions and phenomena created out of the cultural, environmental and aspirational factors prevailing at the time. Capitalism is just one knot in the long thread of human endeavour, emerging as a response to the growing complexity of interrelationships in trade, society and technological knowledge. Yet its social corollary hardly differs from the other social institutions that have arisen in different places and at different times - the partners of capitalism are the age-old issues of inequality and strife.

Recently another ingredient has been added to this pernicious and seemingly unavoidable spin-off: climate change and associated environmental degradation. As our rush towards a fragile future for our descendants continues, politicians and corporate agencies seem to find argument and self-interest, expressed at lavish but irresolute international meetings, more rewarding than meaningful action.

The rest of us might reasonably be expected to ask - is this the best we can do? Given our level of global awareness on matters such as technology, poverty, ecological inter-relatedness and cultural difference, is the best effort that our institutions can make just to fight their own corner of local or national self-interest and lose sight of wider implications? Must we wonder if perhaps it is not institutions that are failing us, but our own natures? Are humans themselves unable to become ideal social beings, or create institutions that look beyond self-interest? Are the liberal, ethical and democratic values that have developed in human consciousness over the millennia all to no avail? If so, then we can hardly feel optimism for our future.

However, it was not with such a pessimistic outlook, but with a sense that change is possible, as long as the vehicles for its expression are available, that Asian Business \& Management ( $A B M$ ) was founded, even as a small voice speaking from a specific perspective. Here, we seek continuously to shed light on the linkages, at both macro and micro level, between issues like the above and the business and management practices pursued in today's world. In contemporary capitalist society, we feel, business is the key institution, with management acting as its function and process - they therefore share 
responsibility for the social and environmental consequences of their action. Business should be responsive and answerable to the macro-reality of this planet and all those living on it, not just to the interests of shareholders and corporate executives. This, we feel, is the only way to assure not just prosperity, but an equitable future.

Diversity embraces more than a calculation of labour and production costs, and $A B M$ always seeks to be a reminder that the complexity of our socioeconomic system can be analysed, and diagnoses of its shortcomings - like financial crises, unemployment, poverty, war and environmental problems are available, if there is willingness to think and act from a broader and longerterm perspective. Quick-patch fixes as we have seen in the 2008 crisis, like any symptom-suppressant medication, may do no more than mask a more serious problem developing behind the scenes. A radical re-conceptualisation of global financial systems will be required if we seek to avoid another global financial crisis surfacing on an even larger scale in the future. In our view, this requires greater democratic and ethical conceptualisation within the spirit of current global capitalism as now practised.

Our eight complete volumes of $A B M$ have aimed to speak towards such an end. In the last year, we have published nineteen articles linking management studies to globalisation, technology, CSR, sustainability, financial crisis, FDI and culture. Theoretically, they explored multinational corporations, national economic systems, foreign subsidiaries, supply chain, knowledge leadership, entry strategies, ownership, manufacturing strategies and entrepreneurship. Geographically, they embraced China, Japan, Korea, India and Asia as a regional unit, though we must always bear in mind that the implications of our published papers, in line with the global context of our contemporary problems, go far beyond our regional focus.

In 2010, our schedule includes two special issues - one on natural environment and business, and the other on the rise of Asian multinationals. In this issue, we present two perspective papers that we are sure readers will find stimulating, even challenging - one on how to conceptualise China's idiosyncratic market economy, and the other on the failure of the libertarian capitalist approach to tackling poverty. Both provide food for thought regarding the meaning of the institutions and ideologies we have created in the contemporary world, and we invite your feedback on them. To this end, we are intending to set up a section on our $A B M$ website for such direct reader input on the research and perspectives put forward in the journal.

To move on to other matters arising over the last year. Our intention to move into on-line submissions was not realised in 2009, but should be in place this year. We have reshuffled our editorial and advisory boards to render a more effective and efficient service for $A B M$ readers and contributors. We look forward to reducing the time taken to review and comment upon submitted 
papers and to becoming more responsive to our audience in general; and we are pleased to add that from this issue, book reviews will once again become a regular feature of the journal.

Finally, inevitably there have been changes in $A B M$ 's key staff, and we would like to send our thanks and best wishes to Ms Susie Tranter, our submissions editor since our inception, who has withdrawn in order to concentrate on her duties at the University of Sheffield, and Ms Megumi Hirose, who has retired from ITEC at Doshisha University to pursue her career with a publishing company in Kyoto. We would like to welcome, as submission and book review editor, Mr Kiyohiko Hasegawa, who will this year be taking up a post with the Department of Peacekeeping Operations at the United Nations.

And of course, we thank those who have contributed their time, despite other demanding commitments, as peer reviewers for our papers; without their help, we could not sustain the quality of this journal.

Harukiyo Hasegawa

General Editor

Doshisha Business School, Kyoto, Japan 